Series A

I. MATHEMATICA

587

\title{
BIHARMONIC MEASURE
}

BY

LEO SARIO

HELSINKI 1974

SUOMALAINEN TIEDEAKATEMIA 
Copyright (C) 1974 by

Academia Scientiarum Fennica

ISSN 0066-1953

ISBN 951.41.0201.0

Communicated 11 Narch 1974

KESKUSKIRJAPAINO

HELSINKI 1974 


\section{Biharmonic measure}

The harmonic Green's function $g$ is known to exist on a Riemannian manifold $R$ if and only if the harmonic measure (1) of the ideal boundary $\beta$ does not reduce to a constant. This measure is a harmonic function on the complement $S_{0}=R-\bar{R}_{0}$ of the closure of a regular region $R_{0}$, with essentially the boundary values 1 on $\partial R_{0}$ and 0 on $\beta$. The biharmonic Green's function $\gamma$ on $R$, with "boundary values» $\gamma=\Delta \gamma=0$ on $\beta$, exists if and only if $\omega \in L^{2}\left(S_{0}\right)$ [22]. In the present paper we ask: can a nonharmonic biharmonic function be introduced on $S_{0}$ with the property that its nondegeneracy characterizes the existence of $\gamma$, in analogy with the nondegeneracy of $\omega$ characterizing the existence of $g$ ? We shall show that this is possible. The function, which we will call the biharmonic measure $\sigma$ of $\beta$, is the limit of biharmonic functions $\sigma_{\Omega}$ on subregions $S_{0} \cap \Omega$ of $S_{0}$, with the $\Omega$ regular subregions of $R$ containing $\bar{R}_{0}$. In contrast with approximating harmonic measures $\omega_{\Omega}$ with $\omega_{\Omega}=1$ on $\partial R_{0}, \quad \omega_{\Omega}=0$ on $\partial \Omega$, the function $\sigma_{\Omega}$, with $\Delta \sigma_{\Omega}=\omega_{\Omega}$, vanishes on the entire boundary of $S_{0} \cap \Omega$, and spans $S_{0} \cap \Omega$ like an arc shaped bridge. As $\Omega$ increases, the height of this arch $\sigma_{\Omega}$ increases, and its limit $\sigma$ as $\Omega$ exhausts $R$ is either an arch spanning $S_{0}$ or else the constant $\infty$. We shall show that the finiteness of $\sigma$ is independent of the choice of $S_{0}$, and we can therefore introduce the class $O_{2}$ of Riemannian manifolds with boundaries of infinite biharmonic measure.

We first explore $O_{\Sigma}$ in its own right. For radial spaces, which play a central role in biharmonic classification theory, we decompose $\sigma$ into its biharmonic, harmonic, quasiharmonic, and constant components. The biharmonic type of $R$ can then be easily tested. In particular, $\sigma<\infty$ if both the biharmonic and harmonic components of $\sigma$ tend to zero as one approaches $\beta$. We use this test to determine the type of a number of fundamental manifolds used in biharmonic classification theory. E.g., for the Poincaré $\mathrm{N}$-ball

The work was sponsored by the U. S. Army Research Office, Grant DA-ARO. 31-124-73-G39, University of California, Los Angeles.

MOS Classification 31B30. 


$$
B_{\alpha}^{N}=\left\{x=\left(x^{1}, \ldots, x^{N}\right),|x|=r, r<1, d s=\left(1-r^{2}\right)^{\alpha}|d x|, \alpha \in \mathbf{R}\right\}
$$

we obtain the following complete characterizations:

$$
B_{\alpha}^{N} \in O_{\Sigma} \Leftrightarrow \begin{cases}\alpha \leq-3 / 2, & N=2, \\ \alpha \notin(-3,1), & N=3, \\ \alpha \geq(N-2)^{-1}, & N>3 .\end{cases}
$$

After this study of $\sigma$ we establish its characteristic property in our original problem:

$$
O_{\Sigma}=O_{T}
$$

where $O_{\Gamma}$ is the class of Riemannian manifolds which do not carry the biharmonic Green's function $\gamma$. In particular, the above values of $\alpha$ exclude $\gamma$ on the Poincaré ball $B_{\alpha}^{v}$.

The role of $O_{\Sigma}$ in general biharmonic classification theory will be discussed in another context.

1. The Laplace-Beltrami operator $\rfloor=d \delta-\delta d$ gives the class $H$ of harmonic functions $h, \Delta h=0$, and the class $H^{2}$ of nonharmonic biharmonic functions $u, \Delta^{2} u=0, \Delta u \neq 0$. Let $R_{0}, \Omega$ be regular subregions of $R$, with $\bar{R}_{0} \subset \Omega$, and set $S_{0}=R-\bar{R}_{0}, \alpha=\partial R_{0}, \beta_{\Omega}=\partial \Omega$. Take $\omega_{\Omega} \in C\left(\bar{S}_{0} \cap \bar{\Omega}\right), \omega_{\Omega} \in H\left(S_{0} \cap \Omega\right), \omega_{\Omega}\left|\alpha=1, \omega_{\Omega}\right| \beta_{\Omega}=0$. The directed limit $\omega=\lim _{\Omega \rightarrow R} \omega_{\Omega}$ is the harmonic measure on $\bar{S}_{0}$ of the ideal boundary $\beta$ of $R$.

We introduce:

Definition. The directed limit

$$
\sigma=\lim _{\Omega \rightarrow R} \sigma_{\Omega}
$$

where

$$
\sigma_{\Omega} \in C\left(\bar{S}_{0} \cap \bar{\Omega}\right) \cap H^{2}\left(S_{0} \cap \Omega\right) . \quad j \sigma_{\Omega}=\omega_{\Omega} . \quad \sigma_{\Omega} x=\sigma_{\Omega} \mid \beta_{\Omega}=0,
$$

is the biharmonic measure of the ideal boundary $\beta$ of the Riemannian manifold $R$.

The limit always exists. In fact. if $g_{S_{0} \cap \Omega}(x, y)$ is the harmonic Green's function on $S_{0} \cap \Omega$ with pole $y$, then

$$
\sigma_{\Omega}(x)=\int_{S_{0} \cap \Omega} g_{S_{\mathrm{c}} \cap \Omega}(x, y) \omega_{\Omega}(y) d y,
$$

with $d y$ the Riemannian volume element at $y$. Since both $g_{S_{0} \cap \Omega}$ and $\omega_{\Omega}$ increase with $\Omega$, so does $\sigma_{\Omega}$, and the limit $\sigma$ exists, finite or infinite. at every $x \in S_{0}$. We shall show in No. 7 that the finiteness is independent of $x$ and of $S_{0}$. 
2. We first study the biharmonic measure $\sigma$ on radial spaces, e.g., the $N$-space and the $N$-ball, each endowed with a radial metric $d s=\lambda(r) d x \quad, \quad r=|x|$. We choose $R_{0}=\left\{r<r_{0}\right\}, \quad S_{0}=\left\{r>r_{0}\right\}$.

Let $Q$ bə the class of quasiharmonic functions $q$, defined by $\Delta q=1$. The space of all radial biharmonic functions $u(r), \Delta^{2} u(r)=0$, is generated by four functions: any $u_{0}(r) \in H^{2}-Q, \Delta u_{0}(r)$, any $q_{0}(r) \in Q$, and the constant 1. Thus every $u(r)$ has a decomposition

$$
u(r)=a u_{0}(r)+b \Delta u_{9}(r)+c q_{0}(r)+d,
$$

which depends on the choice of $u_{0}$ and $q_{0}$. In particular, if the biharmonic measure

$$
\sigma(x)=\sigma(r)=\int_{S_{0}} g_{S_{0}}(x, y) \omega(y) d y
$$

is finite, it has such a decomposition, and $\sigma(x) \rightarrow 0$ as $x \rightarrow \beta$.

Testing of the finiteness of $\sigma$ is facilitated by the following simple criterion:

Lemma. If every radial $u \in H^{2}$ on $S_{0}$ is unbounded, then the biharmonic measure $\sigma$ is infinite. If there exists a function $u_{0}(r) \in H^{2}-Q$ with $u_{0}(r) \rightarrow 0, \Delta u_{0}(r) \rightarrow 0$ as $x \rightarrow \beta$, then $\sigma<\infty$.

Proof. The first part of the Lemma is clear, since $\Delta \sigma=\omega$ entails $\sigma \in H^{2}$. If $u_{0}(r) \rightarrow 0, \Delta u_{0}(r) \rightarrow 0$ as $x \rightarrow \beta$ for some $u_{0} \in H^{2}-Q$, then $a=1 / \Delta u_{0}\left(r_{0}\right)$ gives $\Delta\left(a u_{0}\right)=\omega$, and $b=-a u_{0}\left(r_{0}\right) / \Delta u_{0}\left(r_{0}\right)$ provides us with the function $\sigma=a u_{0}+b \Delta u_{0}$ which has all the properties required of the biharmonic measure.

3. For our first radial space we take the Euclidean $N^{N}$-space $E^{N}$. The type distinction is here fascinatingly simple:

Theorem 1. The ideal boundary of $E^{x}$ has a finite biharmonic measure if and only if $\mathrm{T}>4$.

Proof. Let us first construct $\sigma$ for $N>4$ by means of the definition $\sigma=\lim _{\Omega \rightarrow R} \sigma_{\Omega} . \quad$ Choose $r_{0}=1, \quad \varrho>1, \Omega=\{r<\varrho\}$, and write $\sigma_{\varrho}$ for $\sigma_{\Omega}$. For the decomposition

$$
\sigma_{g}=a_{g} u_{0}(r) \div b_{\underline{u}}-u_{0}(r)+c_{o} q_{0}(r)+d_{\underline{g}}
$$

we take

$$
\sigma_{Q}=a_{Q} r^{-N+4}+b_{Q} r^{-x^{-2}}-c_{Q} r^{2}+d_{Q},
$$

where we have absorbed in $b_{g}$ the constant $[2(N-4)]^{-1}$ from $\Delta r^{-x_{-4}}=$ $2(N-4) r^{-N+2}$, and in $c_{o}$ the constant $-(2 N)^{-1}$ from $\Delta r^{2}=-2 N$. By the definition of $\sigma_{o}$, 


$$
\left\{\begin{array}{l}
\sigma_{\varrho}(1)=a_{\varrho}+b_{\varrho}+c_{\varrho}+d_{\varrho}=0 \\
\sigma_{\varrho}(\varrho)=a_{\varrho} \varrho^{-N+4}+b_{\varrho} \varrho^{-N+2}+c_{\varrho} \varrho^{2}+d_{\varrho}=0 \\
\Delta \sigma_{\varrho}(1)=2(N-4) a_{\varrho}-2 N c_{\varrho}=1 \\
\Delta \sigma_{\varrho}(\varrho)=2(N-4) a_{\varrho} \varrho^{-N+2}-2 N c_{\varrho}=0 .
\end{array}\right.
$$

From the third and fourth equations we obtain

$$
\begin{aligned}
& a_{\varrho}=\left[2(N-4)\left(1-\varrho^{-N+2}\right)\right]^{-1} . \\
& c_{\varrho}=\varrho^{-N+2}\left[2 N\left(1-\varrho^{-N \div 2}\right)\right]^{-1} .
\end{aligned}
$$

If $a, b, c, d$ are the limits of $a_{\varrho}, b_{\varrho}, c_{\varrho}, d_{\varrho}$ as $\varrho \rightarrow \infty$, then

$$
a=[2(N-4)]^{-1}, c=0 .
$$

The first and second equations above then give

$$
\begin{gathered}
b=\lim _{g \rightarrow \infty}\left\{-\left(1-e^{-x+2}\right)^{-1}\left[a_{0}\left(1-e^{-x-4}\right)-c_{g}\left(1-Q^{2}\right)\right]\right\}=-[2(X-4)]^{-1}, \\
d=\lim _{g \rightarrow \infty}\left[-\left(a_{0,}-b_{0}-c_{0,1}\right)\right]=0 .
\end{gathered}
$$

Thus we have the explicit expression

$$
\sigma(r)=[2(N-4)]^{-1}\left(r^{-N \div 4}-r^{-N \div 2}\right)
$$

for the biharmonic measure of the ideal boundary of $E^{N}$ for $N>4$.

4. We now deduce the same result for $N^{r}>4$ using the Lemma and its proof. For $u_{0}(r)=r^{-N+4}, \Delta u_{0}(r)=2(N-4) r^{-N+2}$, we have

$$
a=[\underline{2}(X-4)]^{-1} r_{0}^{Y-2}, b=-[2(X-4)]^{-2} r_{0}^{Y},
$$

and for $r_{0}=1$ the function $\left.\sigma=a u_{0}-b\right\lrcorner_{u_{0}}$ is the biharmonic measure $[2(N-4)]^{-1}\left(r^{-x_{-4}}-r^{-Y_{-2}}\right)$.

To prove that $\sigma=x$ for $x \leq 4$, we use the representation for $\sigma<x$

$$
\sigma(r)= \begin{cases}a r^{2} \log r-b \log r-c r^{2}-d: \quad Y=2, \\ a r+b r^{-1}-c r^{2}-d . & X=3 \\ a \log r+b r^{-2} \div c r^{2}-d . & N=4 .\end{cases}
$$

For $N=2$, this is unbounded unless $a=b=c=0$, and $\sigma$ is constant. For $N=3$ or 4 , it is unbounded unless $a=c=0$ and $\sigma$ is harmonic. However, by $\Delta \sigma=\omega, \sigma$ cannot be harmonic, a contradiction. The proof of Theorem 1 is complete.

5. Can the biharmonic measure of the ideal boundary of $E^{N}$ be made finite even for $N \leq 4$ if we "shrink» or »expand" the boundary by replacing 
the Euclidean metric $d s=|d x|$ by the metric $\left(1+r^{2}\right)^{\alpha}|d x|$, with $\alpha$ a sufficiently small or large constant? Denote the resulting space by $R_{\alpha}^{N}$. The answer is perhaps somewhat unexpected:

Theorem 2. The biharmonic measure $\sigma$ of the ideal boundary of $R_{\alpha}^{N}$ is infinite for all $\alpha$ if $N \leq 4$. For $N>4, \sigma$ is infinite if and only if $\alpha \leq-\frac{1}{2}$.

Proof. An explicit construction of $\sigma$ as the limit of $\sigma_{\Omega}$, as in No. 3, is now not possible, and we make use of the Lemma. We know that $\sigma$ has the form

$$
\sigma(r)=a u_{0}(r)+b \Delta u_{0}(r)+c q_{0}(r)+d .
$$

First we shall find bounded functions $u_{0}, \Delta u_{0}$ for $N>4$. Choose again $S_{0}=\{r>1\}$. For $h(r) \in H\left(S_{0}\right)$,

$$
\begin{aligned}
\Delta h(r) & =-r^{-N+1}\left(1+r^{2}\right)^{-N \alpha}\left[r^{N-1}\left(1+r^{2}\right)^{(N-2) \alpha} h^{\prime}(r)\right]^{\prime}=0, \\
h(r) & =c \int^{r} r^{-N+1}\left(1+r^{2}\right)^{-(N-2) \alpha} d r \sim c \int^{r} r^{-N+1-2(N-2) \alpha} d r \\
& =\left\{\begin{array}{l}
a+b r^{-(N-2)(1+2 \alpha)} \text { if } N>2 \text { and } x \neq-\frac{1}{2}, \\
a+b \log r \text { if } N=2 \text { or } x=-\frac{1}{2} .
\end{array}\right.
\end{aligned}
$$

Thus $h(r)$ belongs to the family $B$ of bounded functions if and only if $N>2, \alpha>-\frac{1}{2}$, an assumption we shall make for the present. Here and later we disregard irrelevant multiplicative and additive constants, and we choose an $h_{0}$ with

$$
h_{0}(r) \sim r^{-(N-2)(1+2 \alpha)},
$$

which $\rightarrow 0$ as $r \rightarrow \infty$ if $N>2, \alpha>-\frac{1}{2}$.

For $\Delta u(r)=h_{0}(r)$, we obtain

$$
\left[r^{Y-1}\left(1+r^{2}\right)^{(Y-2) x} u^{\prime}(r)\right]^{\prime} \sim r^{1+4 x} .
$$

Accordingly, in view of $x=-\frac{1}{2}$. we have [] $r^{2-4 x}$, and, again by virtue of $\alpha \neq-\frac{1}{2}$, we can take $u_{0}$ with

$$
u_{0}(r) \sim r^{-(x-1)(1-2 x)} .
$$

This $\rightarrow 0$ as $r \rightarrow \infty$ if $-2(N-4) x<N-4$, which in turn, under our assumption $\alpha>-\frac{1}{2}$, holds if and only if $Y>4$. By the Lemma, we conclude that $\sigma<\infty$ if $N>4, x>-\frac{1}{2}$.

In the discussion of the case $\sigma=\infty$, the nonuniqueness of the generators $u_{0}, \Delta u_{0}, q_{0}$ (and 1) makes it necessary to consider the unboundedness of all four components of $\sigma(r) \in H^{2}$. For $\Delta q(r)=1$, we obtain 


$$
\left[r^{N-1}\left(1+r^{2}\right)^{(N-2) \alpha} q^{\prime}(r)\right]^{\prime} \sim r^{N-1+2 N \alpha}
$$

and therefore

$$
q^{\prime}(r) \sim \begin{cases}r^{1+4 \alpha}, & \alpha \neq-\frac{1}{2} \\ r^{-1} \log r, & \alpha=-\frac{1}{2}\end{cases}
$$

We choose

$$
q_{0}(r) \sim \begin{cases}r^{2+4 x}, & x \neq-\frac{1}{2}, \\ (\log r)^{2}, & x=-\frac{1}{2} .\end{cases}
$$

For $N=2$ and any $\alpha, h_{0}(r) \sim \log r$, and $u^{\prime}(r)$ satisfies

$$
\left[r u^{\prime}(r)\right]^{\prime} \sim r^{1+4 \alpha} \log r,[] \sim r^{2+4 \alpha} \log r, u^{\prime}(r) \sim r^{1+4 \alpha} \log r,
$$

so that we can take

$$
u_{0}(r) \sim\left\{\begin{array}{l}
r^{2+4 \alpha} \log r, \quad \chi \neq-\frac{1}{2}, \\
(\log r)^{2}, \quad \backslash=-\frac{1}{2} .
\end{array}\right.
$$

Therefore,

$$
\sigma(r) \sim\left\{\begin{array}{l}
a r^{2+4 \alpha} \log r+b \log r+c r^{2-4 x}-d, \quad x \neq-\frac{1}{2} \\
a(\log r)^{2}+b \log r+d, \quad x=-\frac{1}{2} .
\end{array}\right.
$$

By virtue of $\Delta \sigma=\omega$, we have $a \neq 0, b \neq 0$, hence $\sigma \notin B$, and $\sigma=\infty$. For $N=3, x \neq-\frac{1}{2}$,

$$
h_{0}(r) \sim r^{-1-2 \alpha}, u_{0}(r) \sim r^{1+2 \alpha}, q_{0}(r) \sim r^{2+4 \alpha} .
$$

Since $a \neq 0$ and hence $b \neq 0$. we have $\sigma \notin B$, hence $\sigma=x$.

For $\lambda=3, x=-\frac{1}{2}, h_{0}(r) \sim \log r$ and

$$
\begin{aligned}
& {\left[r^{2}\left(1+r^{2}\right)^{-12} u^{\prime}(r)\right]^{\prime} \sim r^{2}\left(1-r^{2}\right)^{-3}{ }^{2} \log r \sim r^{-1} \log r,} \\
& {[] \sim(\log r)^{2}, \quad u^{\prime}(r) \sim r^{-1}(\log r)^{2} . \quad u_{0}(r) \sim(\log r)^{3} .}
\end{aligned}
$$

Since $q_{0}(r) \sim(\log r)^{2}$, we have $\sigma(r) \sim(\log r)^{3} \notin B$, hence $\sigma=x$.

For $N=4, \alpha \neq-\frac{1}{2}, h_{0}(r) \sim r^{-2-4 x}$.

$$
\left[r^{3}\left(1+r^{2}\right)^{2 \alpha} u^{\prime}(r)\right]^{\prime} \sim r^{1-4 \chi} . \quad u^{\prime}(r)-r^{-1}, \quad u_{0}(r) \sim \log r .
$$

In view of $q_{0}(r) \sim r^{2+4 \alpha}, \sigma(r)$ grows at least as rapidly as $\log r$, hence $\sigma=\infty$.

For $N=4, \alpha=-\frac{1}{2}, h_{0}(r) \sim \log r$.

$$
\left[r^{3}\left(1+r^{2}\right)^{-1} u^{\prime}(r)\right]^{\prime} \sim r^{-1} \log r, u^{\prime}(r) \sim r^{-1}(\log r)^{2}, \quad u_{0}(r) \sim(\log r)^{3} .
$$

Since $q_{0}(r) \sim(\log r)^{2}$, we have $\sigma(r) \sim(\log r)^{3} \notin B$, hence $\sigma=\infty$. We have proved that $\sigma=\infty$ for $N \leq 4$, all $\lambda$. 
For $N>4, a<-\frac{1}{2}$.

$$
h_{0}(r) \sim r^{-(N-2)(1+2 x)}, \quad u_{0}(r) \sim r^{-(N-4)(1+2 \alpha)}, \quad q_{0}(r) \sim r^{2+4 x} .
$$

Therefore, $\sigma \sim r^{\beta}$, with

$$
\beta \geq \min [-(N-2)(1+2 x),-(N-4)(1+2 \alpha)] .
$$

The two quantities are both positive for $\alpha<-\frac{1}{2}$, so that $\sigma \notin B$, hence $\sigma=\infty$.

For $N>4, \alpha=-\frac{1}{2}, h_{0}(r) \sim \log r$, $\left[r^{N-1}\left(1+r^{2}\right)^{-(N-2) / 2} u^{\prime}(r)\right]^{\prime} \sim r^{-1} \log r, u^{\prime}(r) \sim r^{-1}(\log r)^{2}, u_{0}(r) \sim(\log r)^{3}$, and $q_{0}(r) \sim(\log r)^{2}$. Consequently $\sigma(r) \sim(\log r)^{3} \notin B$, and $\sigma=\infty$.

We have shown that $\sigma=\infty$ for $N>4, \alpha \leq-\frac{1}{2}$. The proof of Theorem 2 is complete.

6. Next we consider the Poincaré $N$-ball $B_{\alpha}^{N}$, which plays an important role in general biharmonic classification theory. By definition,

$$
B_{\alpha}^{N}=\left\{x=\left(x^{1}, \ldots, x^{-}\right), x=r, r<1, d s=\left(1-r^{2}\right)^{\alpha} d x, \alpha \in \mathbf{R}\right\} .
$$

We shall give a complete characterization of the finiteness of $\sigma$ :

Theorem 3. The biharmonic measure of the ideal boundary of the Poincaré ball $B_{\alpha}^{x}$ is finite if and only if

$$
\begin{cases}\alpha>-3 / 2, & N=2 \\ \alpha \in(-3,1), & N=3 \\ \alpha<(N-2)^{-1}, & N>3\end{cases}
$$

Proof. For $h(r) \in H$,

$$
\Delta h(r)=-r^{-N+1}\left(1-r^{2}\right)^{-N_{\alpha}}\left[r^{--1}\left(1-r^{2}\right)^{(N-2) \alpha} h^{\prime}(r)\right]^{\prime}=0,
$$

and we choose

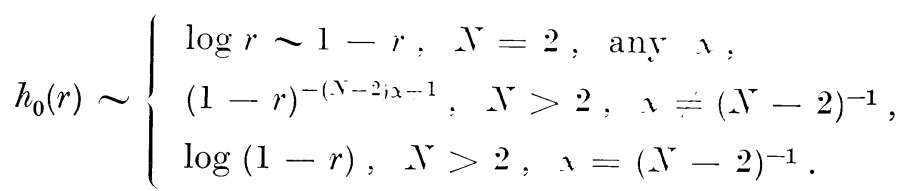

For $\Delta u(r)=h_{0}(r)$

$$
\begin{aligned}
& {\left[r^{N-1}\left(1-r^{2}\right)^{(N-2) \alpha} u^{\prime}(r)\right]^{\prime} \sim(1-r)^{{ }^{\wedge} \chi} h_{0}(r),} \\
& u^{\prime}(r) \sim(1-r)^{-(N-2) \alpha} \int^{r}(1-r)^{{ }^{\nu} x} h_{0}(r) d r .
\end{aligned}
$$


Fore $N=2$, we take

$$
u_{0}(r) \sim \iint^{r}(1-s)^{2 \alpha+1} d s d r \sim\left\{\begin{array}{lr}
\int^{r}(1-r)^{2 \alpha+2} d r \sim(1-r)^{2 \alpha+3}, & x \neq-1, \\
\int^{r} \log (1-r) d r \sim(1-r) \log (1-r), & x=-1, \\
\int^{r}(1-r)^{-1} d r \sim \log (1-r), \lambda=-3 / 2 .
\end{array}\right.
$$

For $N=3$, we obtain successively

$$
\begin{aligned}
& u(r) \sim \begin{cases}\int^{r}(1-r)^{-\alpha} \int^{r}(1-s)^{2 \alpha+1} d s d r, & \alpha \neq 1, \\
\int^{r}(1-r)^{-\alpha} \int^{r}(1-s)^{3} \log (1-s) d s d r, & \alpha=1,\end{cases} \\
& u(r) \sim \begin{cases}\int^{r}(1-r)^{\alpha+2} d r, & x \neq 1,-1, \\
\int^{r}(1-r)^{3} \log (1-r) d r, & x=1, \\
\int^{r}(1-r) \log (1-r) d r, & \alpha=-1,\end{cases} \\
& u_{0}(r) \sim \begin{cases}(1-r)^{\alpha+3}, & \alpha \neq 1,-1,-3, \\
(1-r)^{4} \log (1-r), & x=1 \\
(1-r)^{2} \log (1-r), & x=-1 \\
\log (1-r), & x=-3 .\end{cases}
\end{aligned}
$$

For $N=4$,

$$
u(r) \sim \begin{cases}\int^{r}(1-r)^{-2 x} \int^{r}(1-s)^{2-2-1} d s d r, & x \neq \frac{1}{2}, \\ \int^{r}(1-r)^{-1} \int^{r}(1-s)^{2} \log (1-s) d s d r, & \alpha=\frac{1}{2},\end{cases}
$$




$$
u_{0}(r) \sim \begin{cases}(1-r)^{3}, & x \neq \frac{1}{2},-1 \\ (1-r)^{3} \log (1-r), & x=\frac{1}{2} \\ (1-r)^{3} \log (1-r), & x=-1\end{cases}
$$

For $N>4$,

$$
u(r) \sim \begin{cases}\int^{r}(1-r)^{-(N-2) \alpha} \int^{r}(1-s)^{2 \alpha+1} d s d r, & x \neq(N-2)^{-1}, \\ \int^{r}(1-r)^{-1} \int^{r}(1-s)^{Y_{(Y(X-2)} \log (1-s) d s d r,}, x=(N-2)^{-1},\end{cases}
$$

$u_{0}(r) \sim \begin{cases}(1-r)^{-(N-4) \alpha+3}, & \alpha \neq 3(N-4)^{-1},(N-2)^{-1},-1, \\ \log (1-r), & x=3(N-4)^{-1}, \\ (1-r)^{(2 N-2) /(N-2)} \log (1-r), & x=(N-2)^{-1}, \\ (1-r)^{N-1} \log (1-r), & x=-1 .\end{cases}$

For $\Delta q(r)=1$,

$$
\begin{gathered}
{\left[r^{N-1}\left(1-r^{2}\right)^{(-N-2) x} q^{\prime}(r)\right]^{\prime} \sim(1-r)^{-\times x}} \\
q^{\prime}(r) \sim \begin{cases}(1-r)^{2 x-1}, & x \neq-N^{-1}, \\
(1-r)^{(N-2)-v} \log (1-r), & \alpha=-N^{-1} .\end{cases}
\end{gathered}
$$

For $\quad x \geq 2$,

$$
q_{0}(r) \sim \begin{cases}(1-r)^{2 \alpha+2}, & \alpha \neq-N^{-1},-1, \\ (1-r)^{(2 N-2) / N} \log (1-r), & \alpha=-N^{-1}, \\ \log (1-r), & 1=-1 .\end{cases}
$$

As $r \rightarrow 1$,

$$
\begin{aligned}
& h_{0}(r) \rightarrow 0 \text { if } \begin{cases}N=2, & \text { any } x, \\
N>2, & 1<(\Lambda-2)^{-1},\end{cases} \\
& u_{0}(r) \rightarrow 0 \text { if } \begin{cases}N=2, & \alpha>-3 / 2, \\
N=3, & \alpha>-3, \\
N=4, & \text { any } \alpha, \\
N>4, & \alpha<3(N-4)^{-1} .\end{cases}
\end{aligned}
$$

We conclude by the Lemma that

$$
\sigma<\infty \text { if } \begin{cases}N=2, & \alpha>-3 / 2 \\ N=3, & \alpha \in(-3,1) \\ N>3, & \alpha<(N-2)^{-1}\end{cases}
$$

as claimed. 
In preparation for the case $\sigma=\infty$, we observe that

$$
\begin{gathered}
h_{0} \notin B \Leftrightarrow \begin{cases}N=2, & \text { no } \alpha, \\
N>2, & \alpha \geq(N-2)^{-1},\end{cases} \\
u_{0} \notin B \Leftrightarrow \begin{cases}N=2, & \alpha \leq-3 / 2, \\
N=3, & \alpha \leq-3, \\
N=4, & \text { no } \alpha, \\
N>4, & \alpha \geq 3(N-4)^{-1},\end{cases} \\
q_{0} \notin B \Leftrightarrow N \geq 2, \quad \alpha \leq-1 .
\end{gathered}
$$

We have obtained

$$
\left.a u_{0}+b\right\lrcorner u_{0} \notin B \Leftrightarrow \begin{cases}Y=2, & \alpha \leq-3 / 2, \\ Y=3 . & x \notin(-3,1) . \\ Y>3 . & x \geq(X-2)^{-1} .\end{cases}
$$

except that we shall return later to the case $N^{\top}>4, \quad \geq \geq 3\left(X^{-}-4\right)^{-1}$. Here for $N=2$, we have $h_{0} \in B, u_{0} \notin B$, with

$$
u_{0}(r) \sim \begin{cases}(1-r)^{2 \alpha+3}, & \alpha<-3 / 2, \\ \log (1-r), & \alpha=-3 / 2,\end{cases}
$$

whereas

$$
q_{0}(r) \sim(1-r)^{2 x \div 2}, \quad x \leq-3 / 2
$$

Thus the rates of growth of $u_{0}$ and $q_{0}$ are different for $x \leq-32$, and we have $\sigma \notin B$, hence $\sigma=\infty$ as claimed.

For $N=3, h_{0} \notin B, u_{0} \in B$ if $\alpha \geq 1$, with

$$
h_{0}(r) \sim\left\{\begin{array}{l}
(1-r)^{-\alpha+1}, \quad \alpha>1, \\
\log (1-r), \quad \alpha=1,
\end{array}\right.
$$

whereas

$$
q_{0}(r) \sim(1-r)^{2 \alpha+2}, \quad x \geq 1
$$

Thus the rates of growth are different for $1 \geq 1$. hence $\sigma=x$. Moreover, $h_{0} \in B, u_{0} \notin B$ for $\alpha \leq-3$, with

$$
u_{0}(r) \sim \begin{cases}(1-r)^{2-3}, & x<-3 . \\ \log (1-r), & \quad 1=-3\end{cases}
$$

whereas

$$
q_{0}(r) \sim(1-r)^{2 x-2}, \quad x \leq-3 .
$$


The rates of growth are different for $\alpha \leq-3$, hence $\sigma=\infty$.

For $N=4, h_{0} \notin B, u_{0} \in B$ if $\alpha \geq \frac{1}{2}$, with

$$
h_{0}(r) \sim \begin{cases}(1-r)^{-2 \alpha+1}, & \alpha>\frac{1}{2}, \\ \log (1-r), & \alpha=\frac{1}{2},\end{cases}
$$

whereas

$$
q_{0}(r) \sim(1-r)^{2 \alpha+2}, \alpha \geq \frac{1}{2} .
$$

The rates of growth are different for $\alpha \geq \frac{1}{2}$, hence $\sigma=\infty$.

For $N>4, h_{0} \notin B, u_{0} \in B$ if $x \in\left[(N-2)^{-1}, 3(N-4)^{-1}\right]$, with

$$
h_{0}(r) \sim \begin{cases}(1-r)^{-(N-2) \alpha+1}, & \alpha \in\left((N-2)^{-1}, 3(N-4)^{-1}\right), \\ \log (1-r), & x=(N-2)^{-1},\end{cases}
$$

whereas

$$
q_{0}(r) \sim(1-r)^{2 \alpha+2}, \alpha \in\left[(\Lambda-2)^{-1}, 3(N-4)^{-1}\right) .
$$

Thus the rates of growth are different for $x \in\left[(N-2)^{-1}, 3\left(\lambda^{r}-4\right)^{-1}\right)$, hence $\sigma=\infty$. Moreover, $h_{0} \notin B, u_{0} \notin B$ for $\alpha \geq 3\left(N^{-} 4\right)^{-1}$, with

$$
\begin{aligned}
& h_{0}(r) \sim(1-r)^{-(Y-2) x-1} . \quad x>3(X-4)^{-1}, \\
& u_{0}(r) \sim \begin{cases}(1-r)^{-(N-4)_{x}-3}, & \lambda>3(N-4)^{-1}, \\
\log (1-r), & \alpha=3(N-4)^{-1},\end{cases}
\end{aligned}
$$

whereas

$$
q_{0}(r) \sim(1-r)^{2 \alpha+2}, \alpha \geq 3(N-4)^{-1} .
$$

The rates of growth are all different for $\alpha \geq 3(N-4)^{-1}$, hence $\sigma=\infty$.

The proof of Theorem 3 is herewith complete.

7. We proceed to the proof of the fundamental property of $\sigma$ referred to in No. 1. Let $R$ be an arbitrary Riemannian manifold, $R_{0}$ its regular subregion, and $x$ a point of $s_{0}=R-\bar{R}_{0}$.

Proposition. The finiteness of the biharmonic measure $\sigma(x)$ on $\bar{S}_{0}$ is independent of $R_{0}$ and of $x$.

Proof. For any region $G$, let $g_{G}(x, y)$ be the harmonic Green's function on $G$, with pole $y$. Denote the harmonic measure on $S_{0}$ by $\omega$. The biharmonic measure on $S_{0}$ and the biharmonic Green's function on $R$. if they exist, are

$$
\begin{aligned}
\sigma_{S_{0}}(x) & =\int_{S_{0}} g_{S_{0}}(x, y) \omega(y) d y, \\
\gamma(p, q) & =\int_{R} g_{R}(p, y) g_{R}(y, q) d y .
\end{aligned}
$$


We are to prove:

I. If $\sigma_{S_{0}}(x)<\infty$ for some $S_{0}, x \in S_{0}$, then $\gamma(p, q)<\infty$ for any $p, q \in R$.

II. If $\gamma(p, q)<\infty$ for some $p, q \in R$, then $\sigma_{S_{0}}(x)<\infty$ for any $S_{0}, x \in S_{0}$.

Proof of $I$. Given $\sigma_{S_{0}}(x)<\infty$ for some $S_{0}, x \in S_{0}$, choose any $p, q \in R$ and regular subregions $R_{1}, \Omega$ of $R$ with

$$
\bar{R}_{0} \cup x \cup p \cup q \subset R_{1} \subset \bar{R}_{1} \subset \Omega .
$$

Set $\alpha_{0}=\partial R_{0}, \alpha_{1}=\partial R_{1}, \quad \beta_{\Omega}=\partial \Omega, \quad S_{1}=R-\bar{R}_{1}$, and take

$$
\omega_{\Omega} \in C\left(\bar{\Omega} \cap \overline{S_{0}}\right) \cap H\left(\Omega \cap S_{0}\right), \quad \omega_{\Omega} \lambda_{0}=1, \omega_{\Omega} \mid \beta_{\Omega}=0 .
$$

We shall use the following constants:

$$
\begin{aligned}
& m_{1 \Omega}=\min _{y \in \alpha_{1}} g_{S_{0} \cap \Omega}(y, x), \quad M_{1 \Omega}=\max _{y \in \alpha_{1}} g_{S_{0} \cap \Omega}(y, x), \\
& m_{2 \Omega}=\min _{\alpha_{1}} \omega_{\Omega}, \quad M_{2 \Omega}=\max _{\alpha_{1}} \omega_{\Omega}, \\
& m_{3 \Omega}=\min _{y \in \alpha_{1}} g_{\Omega}(y, p), \quad M_{3 \Omega}=\max _{y \in \alpha_{1}} g_{\Omega}(y, p), \\
& m_{4 \Omega}=\min _{y \in \alpha_{1}} g_{\Omega}(y, q), \quad M_{4 \Omega}=\max _{y \in \alpha_{1}} g_{\Omega}(y, q), \\
& m_{i}=\lim _{\Omega \rightarrow R} m_{i \Omega}, \quad M_{i}=\lim _{\Omega \rightarrow R} M_{i \Omega}, i=1,2,3,4, \\
& k_{1}=\frac{M_{3} M_{4}}{m_{1} m_{2}}, k_{2}=\frac{M_{1} M_{2}}{m_{3} m_{4}} .
\end{aligned}
$$

We obtain

$$
\begin{aligned}
& g_{\Omega}(y, p) \leq \frac{M_{3 \Omega}}{m_{1 \Omega}} g_{s_{0} \cap \Omega}(y, x) \text { on } x_{1} \cup \bar{p}_{\Omega} \text {, hence on } \bar{\Omega} \cap \bar{S}_{1}, \\
& g_{R}(y, p) \leq \frac{\mu_{3}}{m_{1}} g_{s}(y, r) \text { on } \bar{S}_{1} \\
& g_{\Omega}(y, q) \leq \frac{\underline{I_{4 \Omega}}}{m_{2 \Omega}}{ }^{\prime \prime \Omega}(y) \text { on } \bar{\Omega} \cap \bar{S}_{1} \text {, } \\
& g_{R}(y, q) \leq \frac{H_{4}}{m_{2}}\left(\cdot(y) \text { on } \bar{S}_{1} .\right.
\end{aligned}
$$

Therefore,

$$
\int_{S_{1}} g_{R}(p, y) g_{R}(y, q) d y=\int_{S_{1}} g_{R}(y, p) g_{R}(y, q) d y
$$




$$
\begin{aligned}
& \leq k_{1} \int_{S_{1}} g_{S_{0}}(y, x) \omega(y) d y \\
& =k_{1} \int_{S_{1}} g_{S_{0}}(x, y) \omega(y) d y \\
& <k_{1} \int_{S_{0}} g_{S_{0}}(x, y) \omega(y) d y<\infty,
\end{aligned}
$$

and a fortiori

$$
\begin{aligned}
\gamma(p, q) & =\int_{R} g_{R}(p, y) g_{R}(y, q) d y \\
& =C_{1}+\int_{s_{1}} g_{R}(p, y) g_{R}(y, q) d y \\
& =C_{1} \div k_{1} \sigma_{s_{0}}(x)<x .
\end{aligned}
$$

Proof of II. Suppose $\gamma(p, q)<\infty$ for some $p, q \in R$. Take any regular region $R_{0}$ and an $x \in S_{0}=R-\bar{R}_{0}$. For $R_{1}, \Omega$ chosen as before,

$$
\begin{gathered}
g_{S_{0} \cap \Omega}(y, x) \leq \frac{M_{1 \Omega}}{m_{3 \Omega}} g_{\Omega}(y, p) \text { on } \alpha_{1} \cup \beta_{\Omega} \text {, hence on } \overline{S_{1} \cap \bar{\Omega},} \\
g_{s_{0}}(y, x) \leq \frac{M_{1}}{m_{3}} g_{R}(y, p) \text { on } \bar{S}_{1}, \\
\omega_{\Omega \Omega}(y) \leq \frac{M_{2 \Omega}}{m_{4 \Omega}} g_{\Omega}(y, q) \text { on } x_{1} \cup \beta_{\Omega} \text {. hence on } \overline{S_{1} \cap \bar{\Omega}} \\
(1) \leq \frac{M_{2}}{m_{4}} g_{R}(y, q) \text { on } \bar{s}_{1} .
\end{gathered}
$$

'Therefore,

$$
\begin{aligned}
\sigma_{S_{0}}(x) & =\int_{S_{0}} g_{S_{0}}(x, y) \omega(y) d y \\
& =C_{2}+\int_{S_{1}} g_{S_{0}}(x, y) \omega(y) d y \\
& \leq C_{2}+k_{2} \int_{S_{1}} g_{R}(y, p) g_{R}(y, q) d y
\end{aligned}
$$




$$
\begin{aligned}
& =C_{2}+k_{2}\left(C_{3}+\int_{R} g_{R}(p, y) g_{R}(y, q) d y\right) \\
& =C_{2}+k_{2}\left(C_{3}+\gamma(p, q)\right)<\infty .
\end{aligned}
$$

8. In view of the Proposition, we may introduce the class of Riemannian manifolds $R$ with ideal boundaries of infinite biharmonic measure

$$
O_{\Sigma}=\{R \sigma=x\} \text {. }
$$

The class of Riemannian manifolds which do not carry biharmonic Green's functions $\gamma$ is denoted by $O_{T}$ (cf. [2-2]). Properties I and II of $\sigma$ and $\gamma$ provide us with our main result:

Theorem 4. $O_{\Sigma}=O_{\Gamma}$.

As a consequence, e.g. the values of $\alpha$ in Theorem 3 characteriza the Poincaré balls in $O_{\Gamma}$. Moreover, known properties of $O_{\Gamma}$ carry over to $O_{\Sigma}$ E.g., parabolicity implies $\sigma=\infty$.

The author is indebted to Professor Cecilia Wang for a careful checking of the manuscript.

A bibliography of recent work in the field is attached.

University of California, Los Angeles

Department of Mathematics

Los Angeles, California 90024 , CSA 


\section{References}

[1] Chung, L., and SARio, L.: Harmonic $L^{p}$ functions and quasiharmonic degeneracy, (to appear).

[2] - - - - Harmonic and quasiharmonic degeneracy of Riemannian manifolds, Tôhoku Math. J., (to appear).

[3] -"- -"- and WAxG, C.: Riemannian manifolds with bounded Dirichlet finite polyharmonic functions. - Ann. Scuola Norm. Sup. Pisa, (to appear).

[4] -»- -"- -"- Quasiharmonic $L^{p}$ functions on Riemannian manifolds, - Ann. Scuola Norm. Sup. Pisa, (to appear).

[5] Hada, D., Sario, L., and Waxg, C.: Dirichlet finite biharmonic functions on the Poincaré $N$-ball. - J. Reine Angew. Math., (to appear).

[6] -»- - - -»- $N$-manifolds carrying bounded but no Dirichlet finite harmonic functions. - Nagoya Math. J. 54 (1974), 1-6.

[7] -"- - - - - Bounded biharmonic functions on the Poincaré N'ball. Kōdai Math. Sem. Rep., (to appear).

[8] Kwox, Y. K., SARIo, L. and Walsh, B.: Behavior of biharmonic functions on Wiener's and Royden's compactifications. - Ann. Inst. Fourier (Grenoble) 21 (1971), $217-226$.

[9] Mirsky, N., Sario, L., and WANG, C.: Bounded polyharmonic functions and the dimension of the manifold. - J. Math. Kyoto Univ. 13 (1973), 529-535.

[10] NAKaI, M., and SARIo, L.: Completeness and function-theoretic degeneracy of Riemannian spaces. - Proc. Nat. Acad. Sci. 57 (1967), $29-31$.

[11] - - - - B Biharmonic classification of Riemannian manifolds. - Bull. Amer. Math. Soc. 77 (1971), 432-436.

[12] - - - - Quasiharmonic classification of Riemannian manifolds. - Proc. Amer. Math. Soc. 31 (1972), 165-169.

[13] - - - - Dirichlet finite biharmonic functions with Dirichlet finite Laplacians. - Math. Z. 122 (1971), 203-216.

[14] - - - - A property of biharmonic functions with Dirichlet finite Laplacians. - Math. Scand. 29 (1971), 307-316.

[15] - "- - - Existence of Dirichlet finite biharmonic functions. - Ann. Acad. Sci. Fenn. A. I. 532 (1973), 1-33.

[16] - - - Existence of bounded biharmonic functions. - J. Reine Angew. Math. 259 (1973), 147-156.

[17] - - - - Existence of bounded Dirichlet finite biharmonic functions. - Ann. Acad. Sci. Fenn. A. I. 505 (1972), 1-12.

[18] - - - - Biharmonic functions on Riemannian manifolds. - Continuum Mechanics and Related Problems of Analysis, Nauka, Moscow, 1972, $329-335$. 
[19] SARIO, L.: Biharmonic and quasiharmonic functions on Riemannian manifolds. Duplicated lecture notes 1968-70, University of California, Los Angeles.

[20] -》- Quasiharmonic degeneracy of Riemannian N-manifolds. - Kōdai Math. Sem. Rep., (to appear).

[21] - - Completeness and existence of bounded biharmonic functions on a Riemannian manifold. - Ann. Inst. Fourier (Grenoble), (to appear).

[22] - - A criterion for the existence of biharmonic Green's functions, (to appear).

[23] - - Biharmonic measure. - Ann. Acad. Sci. Fenn., (to appear).

[24] - - Biharmonic Green's functions and harmonic degeneracy. - J. Math. Kyoto Univ., (to appear).

[25] - - - and Nakar, M., Classification Theory of Riemann Surfaces. - SpringerVerlag, 1970, $446 \mathrm{pp}$.

[26] - - W - WNG, C.: The class of $(p, q)$-biharmonic functions. - Pacific J. Math. 41 (1972), $799-808$.

[27] - - - - Counterexamples in the biharmonic classification of Riemannian 2-manifolds. - Pacific J. Math. 50 (1974), 159-164.

[28] - - - - Generators of the space of bounded biharmonic functions. - Math. Z. 127 (1972), $273-280$.

[29] -»- - - Quasiharmonic functions on the Poincaré N-ball. - Rend. Mat. (4) $6(1973), 1-14$.

[30] -»- - - Riemannian manifolds of dimension $N \geq 4$ without bounded biharmonic functions. - J. London Math. Soc. - (2) T (1974), 635-644.

[31] -»- - - Existence of Dirichlet finite biharmonic functions on the Poincaré 3-ball. - Pacific J. Math. 48 (1973), $267-274$.

[32] -»- -»- Negative quasiharmonic functions. - Tôhoku Math. J. - 26 (1974), $85-93$.

[33] -»- -»- Radial quasiharmonic functions. - Pacific J. Math. 46 (1973), $515-522$.

[34] -»- - - Parabolicity and existence of bounded biharmonic functions. Comm. Math. Helv. 47 (1972), 341-347.

[35] -»- - - Positive harmonic functions and biharmonic degeneracy. - Bull. Amer. Math. Soc. 79 (1973), $182-187$.

[36] - - - - Parabolicity and existence of Dirichlet finite biharmonic functions. - J. London Math. Soc. - (2) 8 (1974), 145-148.

[37] - - - - Harmonic and biharmonic degeneracy. - Kōdai Math. Sem. Rep. $25(1973), 392-396$.

[38] -»- -»- Harmonic L $L^{p}$-functions on Riemannian manifolds. - Kōdai Math. Sem. Rep., (to appear).

[39] -》- -»- and RANGE, M.: Biharmonic projection and decomposition. - Ann. Acad. Sci. Fenn. A. I. 494 (1971), $1-14$.

[40] WANG, C. and SARIO, L.: Polyharmonic classification of Riemannian manifolds. J. Math. Kyoto Lnir. 12 (1972), 129-140. 